\title{
Angiosarcoma of the adrenal gland with concurrent contralateral advanced renal cell carcinoma: A diagnostic and management dilemma
}

\author{
Kaela Gusenbauer;, Vladimir Ruzhynsky, MD, PhD; ${ }^{*}$ Ipshita Kak, MD; ${ }^{\dagger}$ Anthony F. Adili, MBBCh(C); \\ Kyle Giedraitis,; Snezana Popovic, MD, PhD, MSc, FRCPC, ${ }^{\dagger}$ Anil Kapoor, MD, FRCSC ${ }^{*}$
}

*Division of Urology, Department of Surgery, McMaster University, Hamilton, ON; †Department of Pathology and Molecular Medicine, McMaster University, Hamilton, ON

Cite as: Can Urol Assoc J 2015;9(5-6):E302-5. http://dx.doi.org/10.5489/cuaj.2322 Published online May 13, 2015.

\section{Abstract}

Angiosarcoma is a rare high-grade malignant neoplasm with poor clinical outcome and survival rates, occurring most commonly in the skin and soft tissue. It is composed of neoplastic cells that demonstrate endothelial differentiation. The diagnosis of angiosarcoma can be difficult due to its pathohistologic presentation as a poorly differentiated neoplasm with associated secondary changes. We report a case of angiosarcoma of the adrenal gland with concurrent contralateral renal cell carcinoma (RCC) and renal vein thrombus. The presumptive clinical diagnosis was metastatic renal cell carcinoma to the contralateral adrenal gland. Pathohistologic evaluation demonstrated massive hematoma associated with intravascular papillary endothelial hyperplasia (IPEH)-like features. We discuss the pathohistological features used to ascertain a diagnosis of angiosarcoma in the presence of IPEH-like changes and differentiate it from reactive vascular proliferation seen in IPEH (Masson's tumour).

\section{Introduction}

Angiosarcoma is a rare high-grade malignant neoplasm composed of neoplastic cells showing endothelial cell differentiation. ${ }^{1-4}$ It occurs predominantly in the soft tissue and skin, although other sites including the breast, bone, liver and spleen have been reported. ${ }^{3,5,6}$ Angiosarcoma of the adrenal gland is exceedingly rare and only several cases have been reported..$^{2,7}$

The prognosis of patients with angiosarcoma is poor, with 5 -year survival rates ranging from $24 \%$ to $31 \%$. $13,6,7$ Therefore, a timely correct diagnosis is crucial and is based on immunohistochemical confirmation of the endothelial origin since pathohistologic presentation is typically that of a poorly differentiated neoplasm. ${ }^{4,7}$ We report a case of a patient with adrenal angiosarcoma, discuss the diagnostic challenges of angiosarcoma with the presence of massive hematoma and intravascular papillary endothelial hyperplasia-like features, and differentiate angiosarcoma from intravascular papillary endothelial hyperplasia (IPEH)-Masson's tumour. ${ }^{8}$

\section{Case report}

A 67-year-old man with a medical history of tachyarrhythmia, hypertension, Gastroesophageal reflux disease, benign prostatic hyperplasia, and gout was referred for evaluation of a left renal mass and large right adrenal lesion. His physical examination was benign, except for mild right upper quadrant tenderness. A computed tomography (CT) scan revealed a $15.8-\mathrm{cm}$ heterogeneous enhancing right adrenal lesion (Fig. 1, part A). There was also a $4.8-\mathrm{cm}$ heterogeneous enhancing left renal mass with a $3.6-\mathrm{cm}$ left renal vein thrombus (Fig. 1, part A). The radiographic features of the adrenal lesion were consistent with primary adrenal carcinoma or possibly a metastasis from the renal primary. The adrenal mass metabolic evaluation and metastatic workup were negative.

A CT-guided core biopsy of the adrenal lesion showed inconclusive pathohistologic findings, including fragments of fibroadipose tissue, inflammatory cells, and hemorrhage. There was scanty focus of entrapped cells with clear cell morphology, which could represent benign adrenal cortical cells, however metastatic clear cell carcinoma was considered in the differential diagnosis. It was not possible to perform an additional immunohistochemical study to confirm the definitive cell origin, due to the small size of the core biopsy sample.

The findings were presented at our multidisciplinary cancer rounds and the decision was made to proceed with tyrosine kinase inhibitor neoadjuvant therapy. The patient completed two 4-week courses of sunitinib (50 mg oral, daily). The size of the left renal mass decreased to $3.7 \mathrm{~cm}$ after the first course and the thrombus was present only in 


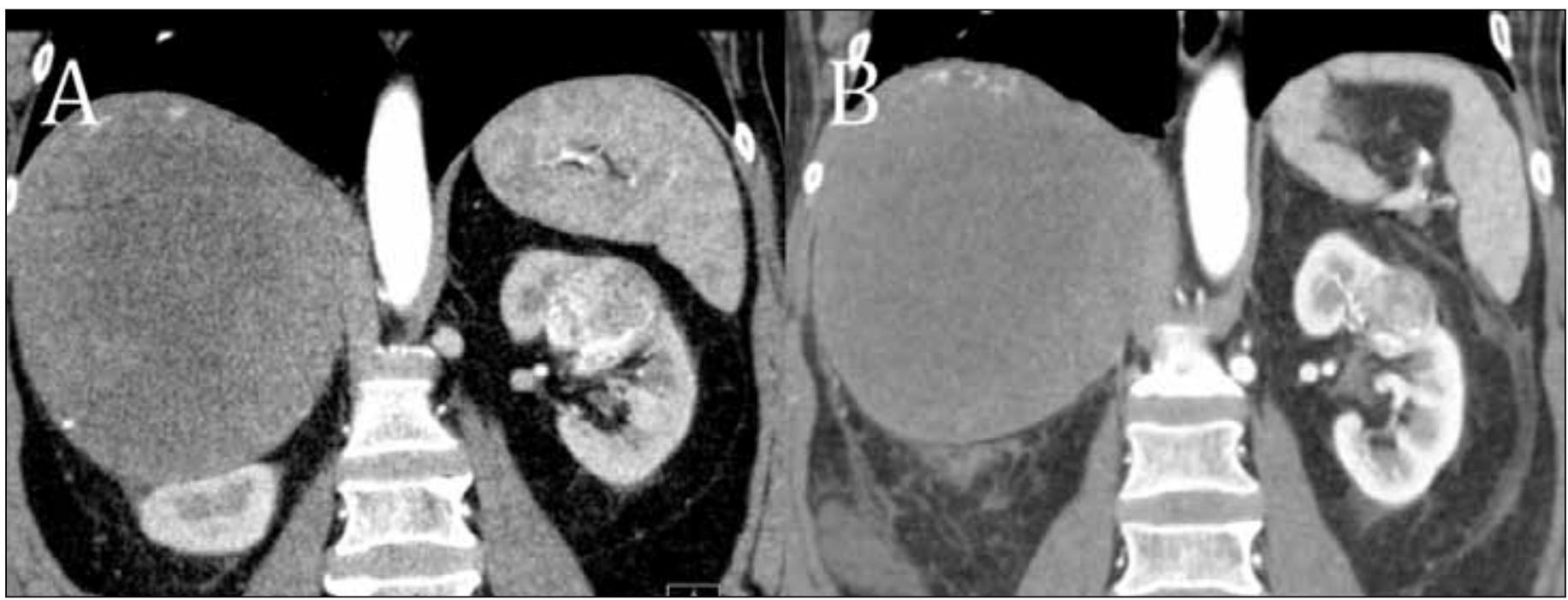

Fig. 1. Contrast-enhanced computed tomography images prior (A) and after (B) the sunitinib treatment demonstrate a large heterogeneously enhancing mass arising from the right adrenal gland. It contains areas of peripheral calcification as well as scattered internal blood products and central necrosis. There is a significant mass-effect on the adjacent organs including inferior displacement of the right kidney. A heterogeneously enhancing lesion is also noted within the upper pole of the left kidney. Note a decreased size of the left renal lesion after the sunitinib therapy.

the uppermost renal segmental vein in keeping with a dramatic treatment response (Fig. 1, part B). The adrenal mass had not changed suggesting that it may not have been a metastatic renal cell carcinoma (RCC).

Faced with this diagnostic dilemma, we found that the left kidney RCC and thrombus responded to sunitinib treatment, while the adrenal mass did not. Therefore, the malignant potential of the adrenal mass was uncertain, and a right adrenalectomy was decided as the next best step in management. The patient underwent a right-sided adrenalectomy 6 weeks after the completion of the second course of sunitinib.

The gross pathological evaluation revealed a $24.5-\mathrm{cm}$ well-circumscribed encapsulated mass with the appearance of a large blood clot and a thick $(1.2 \mathrm{~cm})$ hemorrhagic capsule without evidence of residual adrenal tissue (Fig. 2, part A). Microscopic sections demonstrated massive hematoma surrounded by a fibrous capsule with small foci composed of papillary structures seen at the periphery of the specimen showing papillary endothelial hyperplasia-like features (Fig. 2, part B). The papillary structures were lined with a single layer of endothelial cells forming the occasional anastomosing network with few papillary cores consisting of fibrin or fibroconnective tissue (Fig. 2, part C). Small focal areas arranged in solid sheets of cells were seen close to the fibrous capsule. They were composed of atypical cells showing epithelioid morphology. Infrequent mitotic figures were seen without evidence of atypical mitoses (Fig. 3, parts A, B). Occasional solid foci were intermixed with papillary endothelial hyperplasia-like structures. The endothelial cell differentiation was confirmed by immunostaining (positive CD31 and CD34) (Fig. 3, part C). The lesional cells were negative for epithelial markers and CD10, excluding a possibility of metastatic RCC. Scanty remnant of the adrenal cortical tissue was identified within the capsule (Fig. 3, part

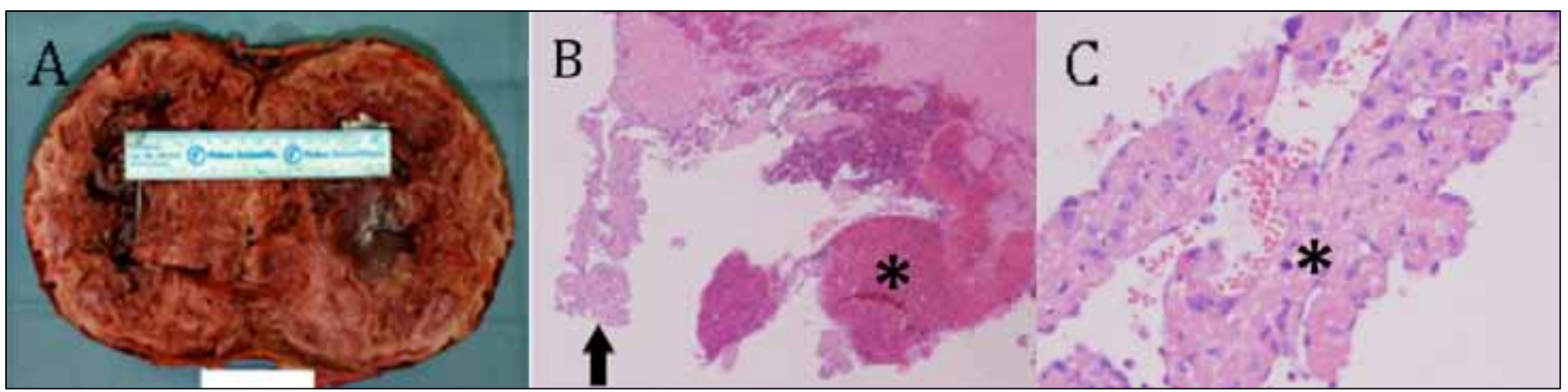

Fig. 2. Gross picture of large encapsulated hemorrhagic adrenal mass with blood clot and variegated heterogeneous appearance (bisected longitudinally). A: Histologic sections of resected adrenal tumour. B: Microscopic section demonstrating widespread areas of hematoma $\left(^{*}\right)$ with papillary structures (arrow) (hematoxylin and eosin stain $[H \& E], \times 20)$. C: Papillary structures $(*)$ lined by a single layer of endothelial cells with occasional fibrin cores $(H \& E, \times 100)$. 


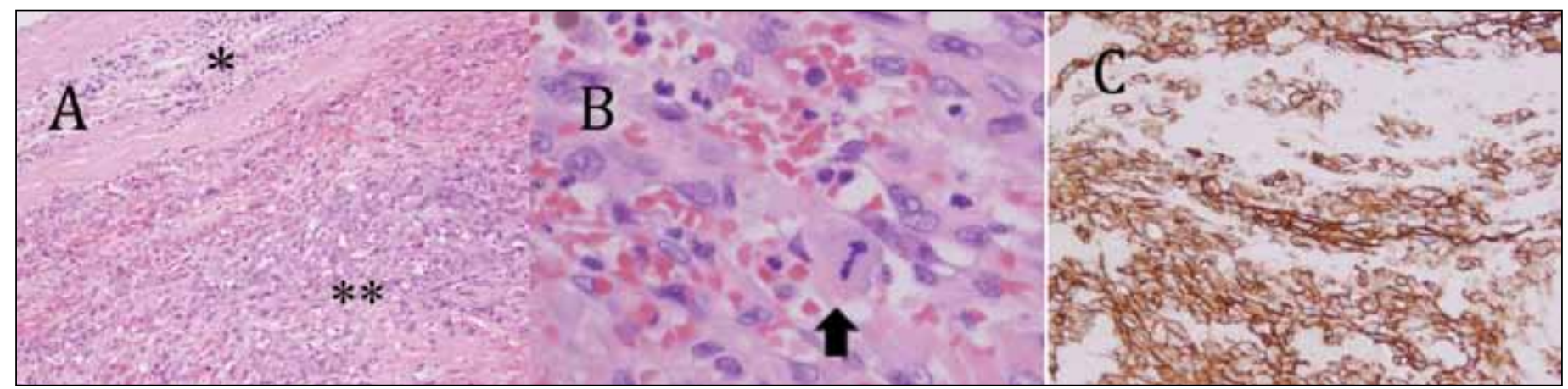

Fig. 3. Histologic sections of adrenal tumour: A. Microscopic section from the periphery of the mass with remnant of adrenal cortical tissue $(*)$ within fibrous capsule and focus of atypical epithelioid cells $\left(^{* *}\right.$ ) (hematoxylin and eosin stain [H\&E] $\times 100$ ). B. Focus of atypical epithelioid cells with occasional mitotic figures (arrow) $(\mathrm{H} \& \mathrm{E}, \times 200)$. C. Immunohistochemistry results showing immunoreactivity for CD31 confirming endothelial origin $(\times 200)$.

A) confirmed by immunostaining (inhibin-positive, vimentinnegative). The specimen was extensively sampled; however, there was no histologic evidence of metastatic RCC; therefore, the possibility of massive adrenal hematoma within metastatic malignancy was excluded. The initial pathohistologic interpretation included papillary endothelial hyperplasia (Masson's tumour) arising in a background of massive hematoma. This reactive process was considered in a differential diagnosis due to lack of frank tumour cell necrosis, marked cellular pleomorphism, relatively low mitotic rate (3 mitoses/10 high power fields), and atypical mitoses. Due to the unusual clinical presentation and the presence of worrisome histologic features, such as small solid foci of atypical epithelioid cells with endothelial differentiation, consultation with the external expert soft tissue pathologist was requested. Despite the Masson-like pathohistologic appearance, based on greater cytologic and nuclear atypia than usually seen in IPEH, a diagnosis of epithelioid angiosarcoma was rendered.

The patient also underwent a left laparoscopic radical nephrectomy with renal vein thrombectomy after the right adrenalectomy. The pathological analysis revealed pT3a clear cell RCC, Fuhrman nuclear grade 2 with clear surgical margin (Fig. 4). The patient is now 1 year post-surgery with negative CT scans with no evidence of recurrent or metastatic disease.

\section{Discussion}

We present a case of a patient with adrenal angiosarcoma and synchronous contralateral RCC. The adrenal angiosarcoma showed unusual histologic presentation with massive hematoma and Masson's tumour-like features. The diagnosis of epithelioid angiosarcoma was confirmed with pathohistological evaluation, which showed endothelial markers, such as CD31, CD34, and factor VIII, as well as a greater degree of cytologic and nuclear atypia than usually seen in Masson's tumour.
The pathogenesis of angiosarcoma remains elusive; however, chronic lymphedema, familial angiodysplasia, prior anabolic steroid therapy, and exposure to vinyl chloride, thorotrast or arsenic have been identified as predisposing factors. The angiosarcomas show upregulation of vascularspecific receptor tyrosine kinases, compared to other types of sarcomas. Furthermore, a high level amplification of MYC is reported in radiation-associated and lymphedema-associated angiosarcoma. ${ }^{2-5,9-11}$ Symptoms of adrenal angiosarcoma are non-specific and range from asymptomatic to significant weight loss, episodic fevers, and weakness. ${ }^{1,5,7,10,11}$

Low incidence rates and non-specific radiologic or histologic features contribute to the difficulty of an angiosarcoma diagnosis. Histologically, it is characterized by solid sheets or nests of epithelioid cells with cellular pleomorphism, increased mitotic activity, eosinophilic cytoplasm, vesicular nuclei, and prominent nucleoli. ${ }^{1-5,7,11,12}$ Furthermore, angiosarcoma tends to have a positive immunoreactivity

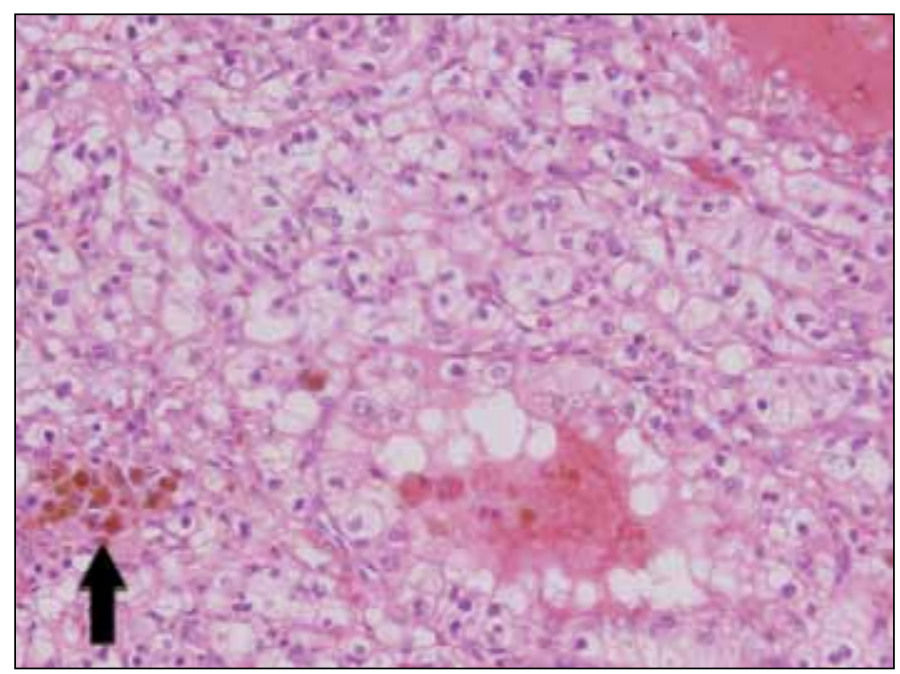

Fig. 4. Microscopic section of the left radical nephrectomy specimen revealing clear cell renal cell carcinoma, Fuhrman nuclear grade 2 with areas of recent and remote hemorrhage and hemosiderin pigment (arrow) (hematoxylin and eosin stain, $\times 200$ ). 
for cytokeratin, a marker of epithelial tumours. ${ }^{2-5,7,11,12}$ Taken together, these factors can contribute to a misdiagnosis of metastatic carcinoma or adrenal carcinoma. However, immunohistochemical staining results using endothelial markers (CD31, CD34, ERG, FLI-1 and factor VIII) can confirm the endothelial differentiation of the neoplasm. ${ }^{3-6,11,12}$

The diagnosis of angiosarcoma can be a challenge due to pathohistologic features mimicking IPEH (Masson's tumour) as seen in the current case. IPEH is a benign, reactive process that cannot be differentiated radiologically from other benign or malignant lesions. ${ }^{13}$ It can mimic malignancy, such as angiosarcoma, in particular in the context of underlying reactive changes related to treatment (sunitinib therapy). IPEH can also occur concomitantly with frank angiosarcoma, making the distinction between these two entities even more challenging, in particular if there is a lack of pathohistologic findings of marked cellular and nuclear pleomorphism, and significant mitotic activity.

Immunohistochemical staining for endothelial markers, such as CD31 and CD34, can be used to differentiate IPEH from non-vascular lesions. ${ }^{14,15}$ However, angiosarcoma also stains positively for these markers. . $^{3,5,6,11,12}$ Angiosarcoma can be differentiated from IPEH based on histological interpretation. Histologic features, such as intraluminal location of IPEH and association with organizing thrombus, may aid in the diagnosis. ${ }^{14}$ Low levels of cellular pleomorphism, necrosis, and mitotic activity are characteristic of IPEH, while in angiosarcoma, these levels tend to be high. ${ }^{7,11,14}$

Prognosis of these two neoplasms are dramatically different. ${ }^{13,14}$ Angiosarcoma is an aggressive high-grade malignancy with a poor prognosis. ${ }^{1,6,7}$ The mortality rate within the first year of diagnosis is over $50 \%{ }^{1}$ Factors that contribute to a poor prognosis include advanced age, increased tumour size, a retroperitoneal primary site, and a proliferative index greater than $10 \% .{ }^{6,12}$ The most common treatment for angiosarcoma is an adrenalectomy, but the presence of metastasis may necessitate adjuvant paclitaxel-based chemotherapy or radiotherapy. ${ }^{2,5-7,12}$ In contrast, with local resection, IPEH can be cured and has a low recurrence rate..$^{13,15}$

\section{Conclusion}

This case demonstrated a RCC of the left kidney with clinically suspected contralateral large adrenal metastasis, which in fact was epithelioid angiosarcoma with Masson's tumourlike histologic features. It illustrates the diagnostic dilemma, and difficult management decisions, which resulted in an excellent surgical outcome. Angiosarcoma can be difficult to diagnose due to the absence of specific radiologic features and histologic features mimicking other neoplastic and non-neoplastic lesions. Immunochemical staining for CD31, CD34, ERG, FLI-1 and factor VIII can confirm the endothelial origin of angiosarcoma. Histologic features, such as the level of cellular pleomorphism and mitotic activity, can be variable, but they are the most important factors used to distinguish angiosarcoma from benign vascular lesions including IPEH. ${ }^{3-7,11,12,14}$ The prognosis and clinical outcome of these two lesions are vastly different and a correct diagnosis is essential to optimize patient outcomes. ${ }^{13,14}$

Competing interests: Dr. Gusenbauer, Dr. Ruzhynsky, Dr. Kak, Dr. Adili, Dr. Giedraitis, and Dr. Popovic declare no competing financial or personal interests. Dr. Kapoor is a member of the Speakers Bureau for Pfizer and Novartis. He has also received grants from Pfizer, GSK, Novartis, and Amgen. He is currently participating in a clinical trial with NCIC, Pfizer, GSK, Novartis, and Amgen.

This paper has been peer-reviewed.

\section{References}

1. Derlin T, Clauditz TS, Habermann CR. Adrenal epithelioid angiosarcoma metastatic to the epicardium: Diagnosis by 18F-FDG PET/CT. Clin Nucl Med 2012;37:914-5. http://dx.doi.org/10.1097/ RLU.0b013e318262afbb

2. Croitoru AG, Klausner AP, McWilliams G, et al. Primary epithelioid angiosarcoma of the adrenal gland. Ann Diagn Pathol 2001;5:300-3. http://dx.doi.org/10.1053/adpa.2001.27917

3. Sung JY, Ahn S, Kim SJ, et al. Angiosarcoma arising within a long-standing cystic lesion of the adrenal gland: A case report. J Clin Oncol 2013;31:e132-6. http://dx.doi.org/10.1200/JC0.2012.44.0800

4. Fletcher CDM, Bridge JA, Hogendoorn PCW, et al. WHO classification of soft tissue and bone. $4 \mathrm{e}$. International Agency for Research on Cancer (IARC), Lyon; 2013:156-7.

5. Stavridis A, Mickovski A, Filipovski V, et al. Epithelioid angiosarcoma of the adrenal gland. Report of a case and review of the literature. Macedon J Med Sci 2010;3:388-94. http://dx.doi.org/10.3889/ MJMS.1857-5773.2010.0119

6. Bacalja J, Ulamec M, Rako D, et al. Adrenal gland hemangiosarcoma in a patient with chronic myeloid leukaemia. Int J Case Rep Med 2013;2013:1-7. http://dx.doi.org/10.5171/2013.784225

7. Pasqual E, Bertolissi F, Grimaldi F, et al. Adrenal angiosarcoma: Report of a case. Surg Today 2002;32:5635. http://dx.doi.org/10.1007/s005950200099

8. Weiss SW, Goldblum JR. Enzinger and Weiss's soft tissue tumors. 5e. Mosby Elsevier; 2007:877-80.

9. Kollmannsberger C, Soulieres D, Wong R, et al. Sunitinib therapy for metastatic renal cell carcinoma: Recommendations for management of side effects. Can Urol Assoc J 2007;1:S41-54.

10. Ferrozzi F, Tognini $G$, Bova D, et al. Hemangiosarcoma of the adrenal glands: CT findings in two cases. Abdom Imaging 2001;26:336-9. http://dx.doi.org/10.1007/s002610000152

11. Wenig BM, Abbondanzo SL, Heffess CS. Epithelioid angiosarcoma of the adrenal glands. A clinicopathologic study of nine cases with a discussion of the implications of finding «epithelial-specific» markers. Am I Surg Pathol 1994;18:62-73. http://dx.doi.org/10.1097/00000478-199401000-00006

12. Hart J, Mandavilli S. Epithelioid angiosarcoma: A brief diagnostic review and differential diagnosis. Arch Pathol Lab Med 2011;135:268-72.

13. Meadows $M C$, Sun $X$, Dardik $M$, et al. Intraabdominal intravascular papillary endothelial hyperplasia (Masson's tumour): A rare and novel cause of gastrointestinal bleeding. Case Rep Gastroenterol 2010;4:124-32. http://dx.doi.org/10.1159/000294148

14. Akdur NC, Donmez M, Gozel S, et al. Intravascular papillary endothelial hyperplasia: Histomorphological and immunohistochemical features. Diagn Pathol 2013;8:167. http://dx.doi.org/10.1186/17461596-8-167

15. Miller TR, Mohan S, Tondon R, et al. Intravascular papillary endothelial hyperplasia of the skull base and intracranial compartment. Clin Neurol Neurosurg 2013;115:2264-7. http://dx.doi.org/10.1016/i. clineuro.2013.07.021

Correspondence: Dr. Anil Kapoor, Professor of Surgery (Urology) McMaster University, 50 Charlton Ave E, Room G344, Hamilton ON L8N 4A6; akapoor@mcmaster.ca 